\title{
Total Knee Arthroplasty Using Virtual Prototyping and Additive Manufacturing
}

DOI: $10.17691 / \mathrm{stm} 2018 \cdot 10.3 .18$

Received November 14, 2017

R. O. Gorbatov, Head of Additive Manufacturing Laboratory, Institute of Traumatology and Orthopedics';

E.E. Malyshev, MD, PhD, Associate Professor, Department of Traumatology, Orthopedics, and Military Surgery named after M.V. Kolokoltsev';

A.D. Romanov, Researcher, Institute of Transportation Systems²;

N.N. Karyakin, MD, DSc, Rector ${ }^{1}$

Privolzhsky Research Medical University, 10/1 Minin and Pozharsky Square, Nizhny Novgorod, 603950, Russia:

${ }^{2}$ Nizhny Novgorod State Technical University named after R.E. Alekseyev, 24 Minin St., Nizhny Novgorod, 603115, Russia

The aim of the study was to evaluate clinical and radiological outcomes of total knee arthroplasty using patient-specific guides to position cutting blocks manufactured by means of virtual prototyping and 3D printing.

Materials and Methods. During the period from 2016 to 2018, six surgeries in total knee arthroplasty were performed at the Institute of Traumatology and Orthopedics of Privolzhsky Research Medical University (Nizhny Novgorod, Russia) using patient-specific precision guides to position cutting blocks. The indication for these surgical operations in patients was the presence of post-traumatic knee joint osteoarthrosis of stage III (according to the classification of N.S. Kosinskaya).

Simulation of surgery on a virtual prototype of the lower limb provided the possibility to carry out quantitative analysis of the following parameters: deformity angles, resection depth, and direction, the size of endoprosthesis components.

Results. The attempt to normalize the lower limb axis proved to be successful in all patients. The anatomical femorotibial angle was $176.1 \pm 2.56^{\circ}$ after surgery, the deviation from the limb axis was $0.45 \pm 0.46^{\circ}$ according to FLFS. In the period from 6 to 12 months after surgery, the average KOOS score was $91.2 \pm 10.1^{\circ}$ for Pain; $83.45 \pm 12.70^{\circ}$ for Daily Physical Activity; $71.1 \pm 15.23^{\circ}$ for Symptoms and Stiffness; $73.10 \pm 16.1^{\circ}$ for Quality of Life. The average range of motion in the knee joint after total knee arthroplasty was $105.2 \pm 10.9^{\circ}$ in flexion and there was full extension. In 4 patients, the data on positioning the cutting blocks corresponded to computer navigation data. In 2 cases, the deviation of the femoral patient-specific precision guide in the frontal plane was $1-3^{\circ}$, in the tibial plane $-2-4^{\circ}$.

The implant size was found to correspond to the results of preoperative planning on computer 3D models in all patients.

Conclusion. Total knee arthroplasty using a virtual three-dimensional prototype of the patient's lower limb and 3D printing allowed achieving good and excellent clinical and radiological results.

Key words: knee arthroplasty; knee joint; 3D technologies; computer modeling; virtual prototyping; 3D printing.

\section{Introduction}

Knee osteoarthritis (gonarthrosis) of stage III-IV (according to the Kellgren and Lawrence classification) is manifested in severe pain syndrome, impaired support and motion function and significant decrease in the patient's quality of life [1]. The incidence of gonarthrosis reaches 99.6 cases per 10,000 adults. In $86 \%$ of cases, this disease occurs in people of working age and leads to disability in $6.5-14.6 \%$ of patients. Knee osteoarthritis accounts for $50.6-54.5 \%$ of all degenerative diseases of the large joints of the lower limbs [2-5].

The first attempts of surgical treatment in patients suffering from gonarthrosis date back to the middle of the $19^{\text {th }}$ century when interposition arthroplasty and knee arthrodesis were first performed [6]. Currently, total knee arthroplasty is one of the most effective methods of surgical treatment, which makes it possible to restore the range of motions and stability in the affected joint, to relieve pain [7]. However, the presence of bone defects, capsular adhesion, scar-adhesions of the anterior femoral muscles, extra-articular deformities, post-traumatic changes in the bone structure lead to unsatisfactory surgical outcomes in more than $17 \%$ of cases [1, 2, 8]. In addition, when the deviation from the normal limb axis is more than $3^{\circ}$, the incidence of aseptic loosening of endoprosthesis components increases to $24 \%$ after 8 years. If the deviation does not exceed $3^{\circ}$, the loosening will be only $3 \%[9,10]$. The use of computer navigation can improve spatial orientation accuracy of knee endoprosthesis components in all planes [9-11]. However, this has a number of disadvantages and the main one is increased operating time. When using computer navigation, diagnosis

Corresponding author: Roman O. Gorbatov, e-mail: gorbatov.ro@yandex.ru 
of anatomical landmarks and the mechanical axis is carried out intraoperatively [1, 12, 13], but navigation may be difficult or impossible in a number of clinical situations, for example, in hip joint ankylosis or severe coxarthrosis, as hip rotation is accompanied by pelvic displacement. Marked ankle deformity also limits the possibility of computer navigation use [1, 3, 13]. Errors can be associated with displacement of probes fixed to the bones during the operation. Computer navigation is impossible in hip arthrodesis, femoral head deformity, complex deformities of the femoral and tibial epiphyses. Its use is difficult in overweight patients [1, 5, 14]. The following results were obtained during computerassisted knee replacement surgery: the mechanical axis of the limb was within the normal range only in $35 \%$ of all operated patients, 1 to $2^{\circ}$ deviation was recorded in $55 \%$. When knee replacement was carried out without computer navigation, $60 \%$ of patients had deviations of up to $4^{\circ}[1,2,12,13]$.

One of the main problems in correct implantation of endoprosthesis components is determining femoral component rotation. Knee joint functioning largely depends on its positioning in three-dimensional space. When internal rotation by $4.7^{\circ}$ occurs, the incidence of pain in the anterior part of the knee increases 5 times, while rotation by $5.5^{\circ}$ results in significant functional limitations developing in the joint. Incorrect rotation of the femoral component by $5^{\circ}$ leads to patellar subluxation and by $10^{\circ}$ to dislocation $[1-3,11,13]$. Besides, increased wear of the endoprosthesis insert is observed when femoral component rotation is incorrect (see the Table).

To avoid aseptic loosening which accounts for $60 \%$ of all revision surgery causes $[3,5,6,15]$, mechanical axis deviation should not exceed $3^{\circ}$, correct rotation should be performed, slope angles of the femoral and tibial components in the sagittal plane should be observed, correct knee ligament balance has to be achieved. When standard knee replacement techniques are used in case of extra-articular knee deformities, there may be errors in the frontal positioning of the components. In some cases, posterior femoral condyle deformity (due to dysplasia, trauma or arthrosis) may result in femoral rotational errors [1-4, 9, 11].

Thus, development of surgical techniques to improve precision of the mechanical axis of limb reconstruction and positioning of endoprosthesis components thereby reducing aseptic loosening of implants remains an urgent problem in modern knee arthroplasty.

The use of patient-specific precision guides (PSG) is an innovative solution to this problem. This technology offers the possibility to perform arthroplasty when there are contraindications to the use of computer navigation and standard guides, to ensure more accurate reconstruction of the lower limb axis and reduce operating room time [5, 13, 14, 16]. Besides, the use of individual guides can improve clinical outcomes after surgery, achieve more accurate rotation of endoprosthesis components compared to computer navigation $[5,14,16]$. Their use is particularly advantageous in the following situations [5, 11-13]:

inflammation or femoral deformity in past medical history, when the use of intramedullary guides is difficult and allows no sufficiently accurate positioning of endoprosthesis components or is associated with increased risk of purulent-inflammatory process;

ipsilateral hip endoprosthesis, when it is desirable to avoid opening the medullary canal;

significant bone defects or massive osteophytes of the posterior femoral condyles complicating correct rotational implantation of the femoral component;

severe movement limitations in the knee joint, when it is technically impossible to perform arthroplasty with computer navigation and the use of standard instrumentation is associated with significant technical difficulties.

The existing methods of knee replacement with the use of PSG include application of 3D-printed patient-specific cutting blocks. However, the use of such technologies without simulation of surgery on the patient's individual virtual lower limb prototype leads to decreased accuracy of patient-specific guide positioning during the surgery and, consequently, to increased number of cases with loosening of endoprosthesis components, decreased number of excellent and good treatment outcomes $[5,14,16]$.

For example, when using the Zimmer PSI patientspecific cutting blocks (Zimmer, Canada) for knee arthroplasty, deviation in the position of the femoral and tibial prosthetic components by more than $3^{\circ}$ compared to the preoperative planning is diagnosed in $3-17 \%$ of cases. The use of GMK MyKnee guides (Medacta International, Switzerland) leads to deviation in the position of endoprosthesis components by more than $3^{\circ}$ from the plan in $12.4 \%$ of cases [11, 12, 17, 18].

The aim of the study was to evaluate clinical and radiological outcomes of total knee arthroplasty using patient-specific guides to position cutting blocks manufactured by means of virtual prototyping and 3D printing.

Characteristics of symptoms in total knee arthroplasty based on femoral component rotation

\begin{tabular}{ll}
\hline $\begin{array}{c}\text { Deviation } \\
\text { from the mechanical axis }\end{array}$ & \multicolumn{1}{c}{ Symptoms } \\
\hline $0-10^{\circ}$ external rotation & No patella problems \\
\hline $1-4^{\circ}$ internal rotation & Pain in the patella, patella lateralization \\
\hline $3-8^{\circ}$ internal rotation & Patellar subluxation, functional limitations in the joint \\
\hline $7-17^{\circ}$ internal rotation & Patellar dislocation and aseptic loosening of endoprosthesis components \\
\hline
\end{tabular}




\section{Materials and Methods}

During the period from 2016 to 2018, six surgical operations in total knee arthroplasty (TKA) were performed at the Institute of Traumatology and Orthopedics of Privolzhsky Research Medical University (Nizhny Novgorod) using patient-specific guides to position cutting blocks manufactured by means of virtual prototyping and $3 \mathrm{D}$ printing. The mean age of patients was $47.67 \pm 9.20$ years. There were 5 women and 1 man among them. Primary knee arthroplasty was performed for post-traumatic gonarthrosis of stage III-IV (according to the Kellgren and Lawrence classification) using Zimmer NexGen implants (Zimmer, Canada) in all cases.

This retrospective study was approved by Ethics Committee of Privolzhsky Research Medical University and compliant with the Declaration of Helsinki (2013). Written informed consent was obtained from every patient.

Initially, all the patients had marked movement limitation in the knee joint. The average amount of flexion was $42.1 \pm 24.5^{\circ}$, extension deficit was $10 \pm 3^{\circ}$. Before surgery, the average KOOS score was $37.21 \pm 3.20$ for Pain; $41.51 \pm 2.60$ for Daily Physical Activity; 43.5 \pm 3.0 for Symptoms and Stiffness; $3.2 \pm 1.3$ for Quality of Life. According to the teleroentgenography (FLFS) data, all patients had severe varus deformity of the lower limb axis $\left(13.0 \pm 10.1^{\circ}\right)$.

The indication to perform TKA was presence of femoral deformity in combination with severe contracture of the knee extensor as the use of the intramedullary guide in this case would not provide sufficient precision of femoral component placement along the limb axis, while computer navigation was technically impossible.

Inflammatory process and femoral deformity in the past history allowed using neither standard intramedullary guide for the hip nor computer navigation due to the increased risk of infectious complications. Presence of severe contracture of the knee extensor and ipsilateral hip endoprosthesis made opening the medullary canal undesirable, the use of computer navigation being impossible.

First, in order to create PSG, CT examination of the affected lower limb was performed from the iliac crest to the talus bone. The data obtained in the form of a series of DICOM files was converted into a 3D model using specialized software. For this purpose, the array of tomographic data was used to create a virtual threedimensional prototype of the lower limb, the cutoff level being empirically determined (according to the Hounsfield scale) to save only bone and cartilage structures. Then, standard software tools were used to simulate positioning of endoprosthesis components taking into account deformities, bone and cartilage defects of the femur and tibia as well as bone density. Next, anatomical structures of the hip, knee and ankle joints were analyzed providing the possibility to assess the initial state in relation to the optimal mechanical axes.
It is characteristic of surgery simulation on a virtual lower limb prototype to take into account the errors in the positioning of endoprosthesis components in all three axes with equal accuracy. The cutting blocks were positioned in the frontal plane according to the conventional technique of limb axis alignment in accordance with the mechanical axis (Mikulicz axis). Rotational alignment of the tibial component was carried out taking into account its orientation to the inner third of the tibial tuberosity. Positioning in the sagittal plane was carried out according to the standard slope of tibial plateau resection with due account for clinical situation particulars and endoprosthesis type. Then, virtual lower limb model was used to carry out topological optimization of the implant attachment points to the deformity area and, if necessary, to adjust the congruence between the implant components and bone surfaces. After that, patient-specific guides for positioning of cutting blocks were created using the methods of hybrid parametric modeling.

On completion of 3D modeling, PSG model was saved in stl format and converted to g-code or $\times 3 \mathrm{~g}$ for subsequent $3 \mathrm{D}$ printing using FDM technology. After patient-specific guides (femoral and tibial components) were sterilized, total knee arthroplasty was performed using a midline incision and a medial parapatellar arthrotomy, positioning PSGs through which the pins were inserted for subsequent setting the position of standard cutting blocks.

Positioning the endoprosthesis components was controlled and the final decision on their implantation was made according to the results of computer navigation using the OrthoPilot system (V. Braun, Aesculap, Germany).

The guides from HIPS material tested for cytotoxicity in the Laboratory of Biotechnology of Privolzhsky Research Medical University were 3D printed on the FDM 3D printer MakerBot Replicator 2 (MakerBot Industries, USA) and Ultimaker 2 extended plus (Ultimaker, Netherlands) with high accuracy of matching (less than $100 \mu \mathrm{m}$ ) computer three-dimensional models. Computer simulation was performed in Autodesk Meshmixer software. After 3D printing, PSG dimensions were measured and compared to three-dimensional model parameters. During the surgery, patient-specific guides corresponding to the planned dimensions with $1 \mathrm{~mm}$ accuracy were used.

The international KOOS scale was used for clinical assessment of patients after knee replacement. The result was assessed as excellent when the sum of scores was 85 and more, the total score from 84 to 70 was assessed as good, from 69 to 60 as satisfactory, less than 60 was regarded as unsatisfactory.

Clinico-radiological examination of patients was performed at the follow-up examination 6 months after the surgery.

Statistical processing of the digital data was performed using Microsoft Office Excel 2010 and 
Statistica 10.0 (StatSoft, USA) software. Descriptive statistics of features included the mean (M) and standard error of the mean $(\mathrm{m})$.

\section{Results}

The overall duration of all stages of manufacturing patient-specific guides for positioning the cutting blocks per one patient was $4 \pm 2$ days. It included computer modeling and simulation $-9 \pm 2 \mathrm{~h}, 3 \mathrm{D}$ printing $-2 \pm 1$ day (the total duration of 3D printer operation $-28.1 \pm 3.4 \mathrm{~h}$ ).

The average intraoperative blood loss was $185 \pm 25 \mathrm{ml}$. Drainage was removed on postoperative day 2 in all cases. The average perioperative blood loss amounted to $241 \pm 50 \mathrm{ml}$. The average operating room time was $40 \pm 15 \mathrm{~min}$, given the deduction of time for probe placement and collection of information necessary for the use of computer navigation. After the surgery, all patients showed significant improvement in the functioning of the operated knee joint and pain relief.

The attempt to normalize the lower limb axis proved to be successful in all patients. The anatomical femorotibial angle was $176.1 \pm 2.56^{\circ}$ after surgery, the deviation from the limb axis was $0.45 \pm 0.46^{\circ}$ according to FLFS. In the period from 6 to 12 months after surgery, the average KOOS score was $91.2 \pm 10.1^{\circ}$ for Pain; $83.45 \pm 12.70^{\circ}$ for Daily Physical Activity; $71.1 \pm 15.23^{\circ}$ for Symptoms and Stiffness; $73.10 \pm 16.1^{\circ}$ for Quality of Life. The average knee range of motion after TKA was $105.2 \pm 10.9^{\circ}$ in flexion and there was full extension. Given the fact that all patients had marked limitation of movements in the knee joint before surgery, the result can be considered as excellent.

In 4 patients, the data on positioning the cutting blocks corresponded to computer navigation data. In 2 cases, the deviation of the femoral $P S G$ in the frontal plane was $1-3^{\circ}$, in the tibial plane $-2-4^{\circ}$.

The implant size was found to correspond to the results of preoperative planning on computer 3D models in all patients. However, it was necessary to reduce the femoral component of the endoprosthesis in one patient to achieve the balance of flexion and extension intervals.

Here is a clinical example.

Patient E., 62 years old.

Complaints about pain in both knee joints, the pain being more severe on the right. Impaired function of support and motion in the right lower limb.

Past history of the disease: pain in the right knee joint appeared about 2 years ago, gradually increased to appear at rest. Currently, conservative therapy brings no significant improvement, the patient has been hospitalized for surgical treatment.

Local status: the patient moves around the department without additional support, limping on the right lower limb. The skin is clean, has normal color, with no visible breaches of integrity. On palpation, there is pain in the right knee joint. Movements in the hip joint are not limited. Active and passive movements in the right knee joint are painful and limited: flexion $-50^{\circ}$, extension deficit $-15^{\circ}$. It has been found during linear measurement of lower limb lengths that $D=L$. There are no skin sensitivity disorders in the hip and tibia. Movements in the ankle are within normal limits. Varus deformity of the right lower limb is diagnosed. There is crunching and clicking sound during right knee joint motion. Floating patella symptom is negative. Visually, there is a slight swelling of the right knee joint. No vascular disorders have been revealed.

According to radiographic data, post-traumatic rightsided gonarthrosis of stage III with varus deformity has been diagnosed (Figure 1).

KOOS assessment: Pain - 25, Symptoms and Stiffness - 30, Daily Physical Activity - 25, Quality of Life -15.

The virtual prototype of the right lower limb is created using CT data (Figure 2).

Hybrid parametric modeling and topology optimization (Figure 3) make it possible to create computer models of patient-specific guides for positioning the cutting block pins (Figure 4) which are later manufactured on FDM 3D printer.

After that, total arthroplasty of the right knee joint is performed with Zimmer NexGen LPS prosthesis (Zimmer, Canada) using patient-specific guides for
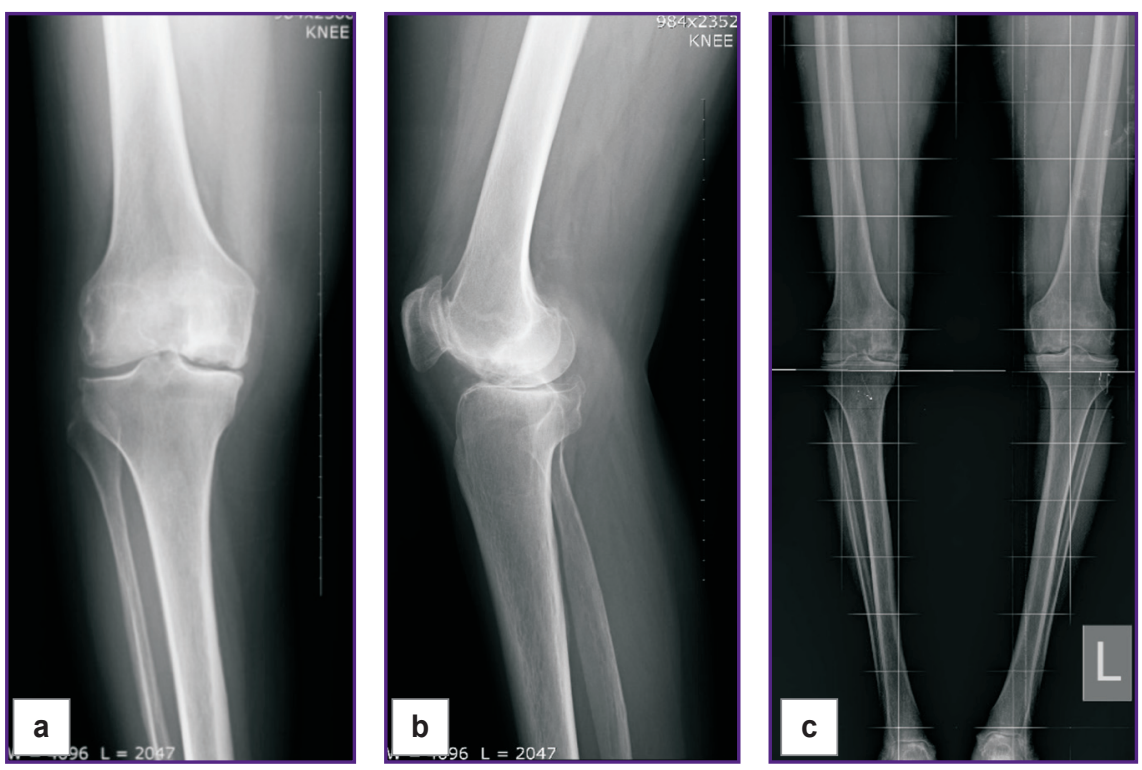

Figure 1. X-ray pictures of patient E., 62 years:

(a) anterior-posterior view; (b) lateral view; (c) X-ray picture of the lower limbs along the length (teleroentgenogram) 


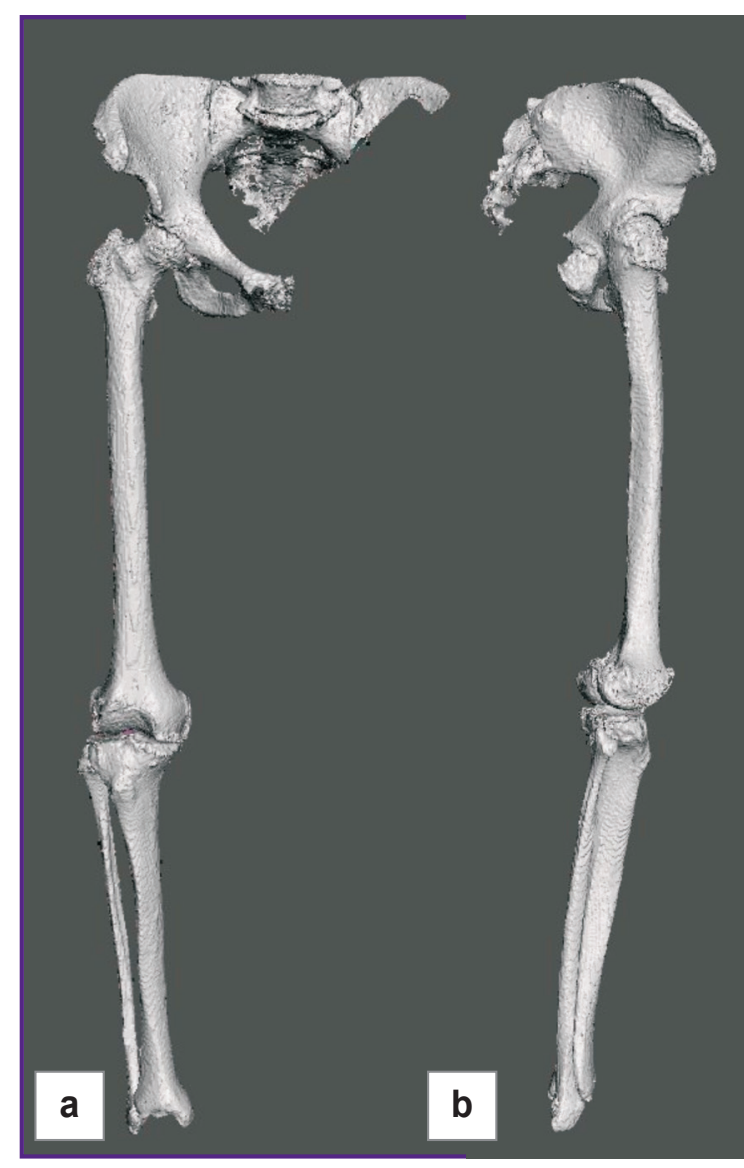

Figure 2. Photo of virtual right lower limb prototype:

(a) frontal view (external rotation projection of the right lower limb); (b) lateral view

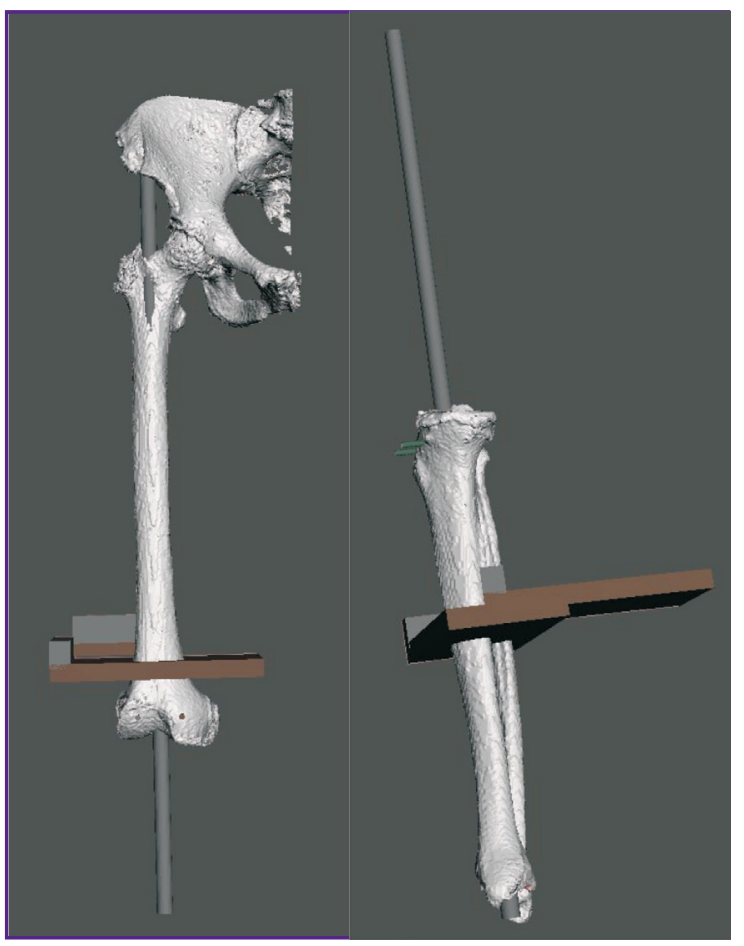

Figure 3. Stages of computer modeling

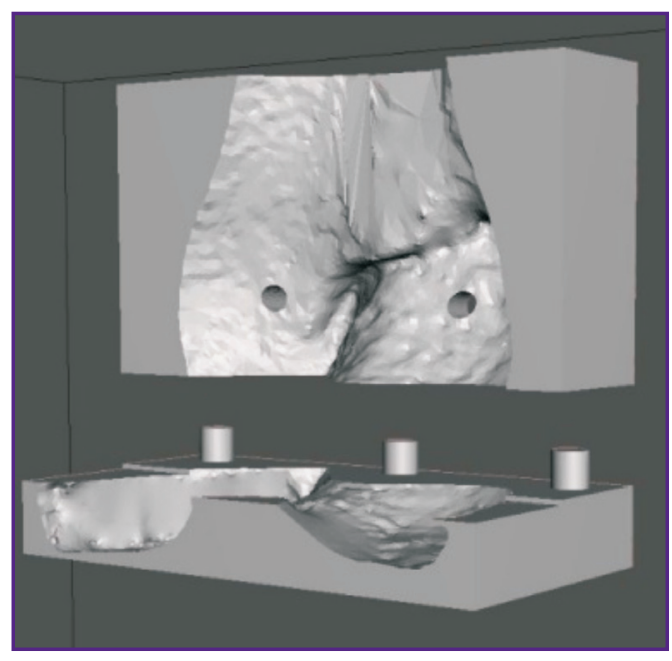

Figure 4. Computer model of a patient-specific guide for positioning the pins of the femoral cutting block of the endoprosthesis

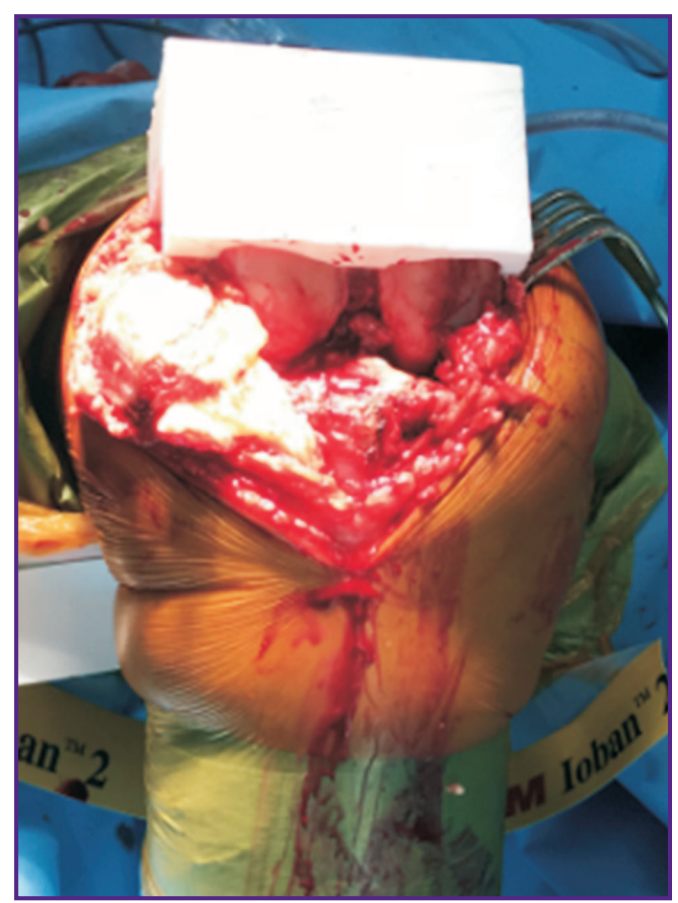

Figure 5. Intraoperative photo of the stage of placing patient-specific guides for positioning the pins of cutting blocks

cutting block pins manufactured according to the developed technology. The lower limb axis is normalized (Figures 5, 6).

During follow-up examination after 6 months, the patient has no complaints. The range of motion in the right knee joint: flexion - $110^{\circ}$, full extension. The patient moves without the means of additional support. KOOS scores: Pain - 100, Symptoms and Stiffness 71, Daily Physical Activity - 77, Quality of Life - 75. 


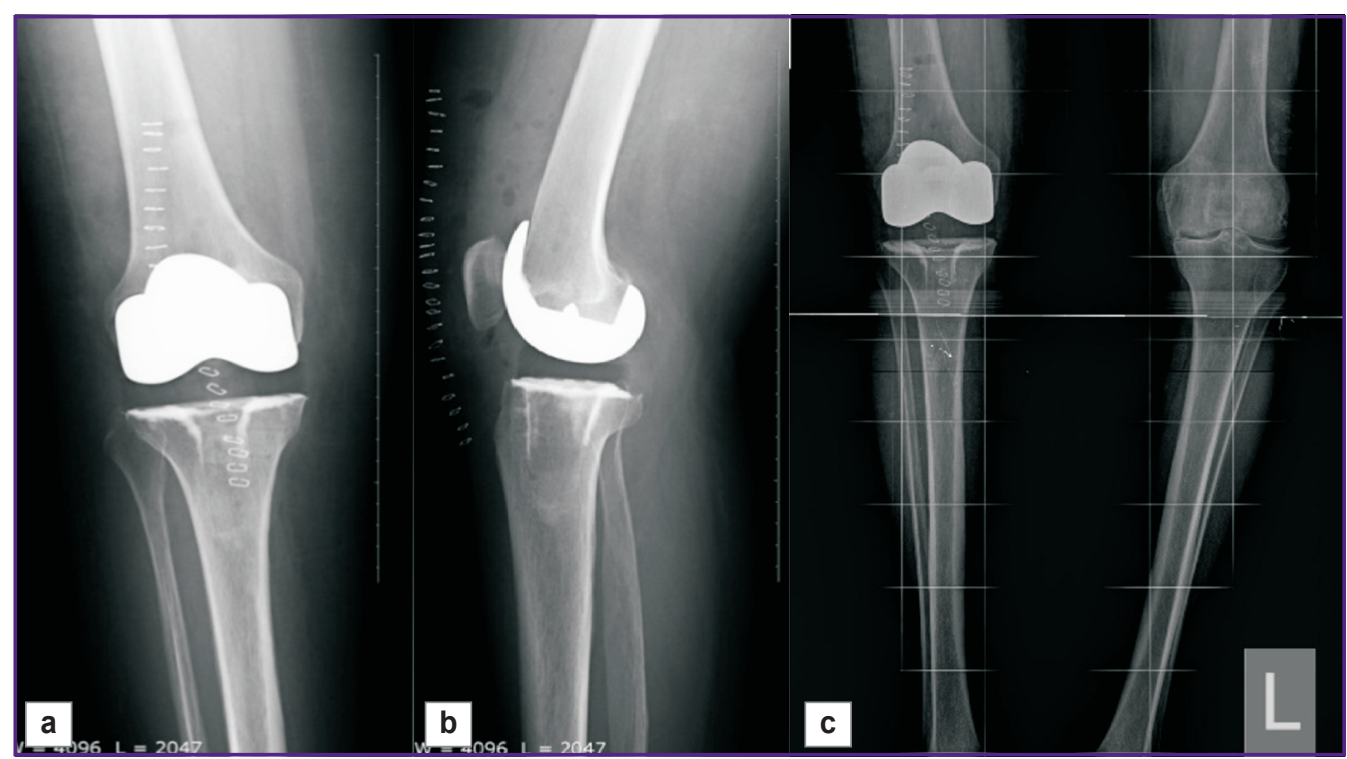

Figure 6. X-ray pictures after right total knee arthroplasty using patient-specific guides:

(a) anterior-posterior view; (b) lateral view; (c) X-ray picture of the lower limbs along the length (teleroentgenogram)

\section{Discussion}

Computer preoperative planning and manufacturing of pin guides on a 3D printer using virtual prototyping offers the possibility to carry out quantitative analysis of parameters: deformity angles, resection thickness, resection amount and direction, dimensions of prosthetic components. However, the use of this knee replacement technique requires excellent knowledge of anatomy by both surgeon and IT-specialist, also it is imperative for the operator to have wide experience in performing arthroplasty with various navigation systems.

Similarly to study [10], we have achieved significant improvement in the functional outcomes of patients after surgery, which confirms clinical efficacy of this technique.

In contrast to works [16] and [13] providing the information on 29 and $37 \%$ of cases reporting more than $3^{\circ}$ deviations from the frontal plane mechanical axis of the lower limb when using PSG, we have observed such a deviation only in 1 of 6 patients. In our opinion, reduction in the number of unsatisfactory results is associated with applying the techniques of hybrid parametric modeling and topological optimization of guides in accordance with anatomical landmarks of three-dimensional computer model of the entire lower limb.

Compared to the results of work [18], we have found no significant reduction in blood loss when using PSG. In our opinion, this is associated with the use of bone autoplasty for closing the hole in the femur, resulting from the use of intramedullary guide, which provides reduction of blood loss from the medullary canal.

We have revealed no operating room time reduction in total knee arthroplasty using PSG in contrast to the data provided in works [11-13].
Similarly to works [19] and [20], it has been established that one of the advantages of PSG use is the precision of rotational positioning of the femoral prosthetic component, which is particularly important and practically significant in cases of severe knee joint contractures, limited intraoperative visualization of both posterior condylar line and supracondylar one.

The implant sizes have been found to match the results of preoperative planning on computer 3D models in all patients, which corresponds to the data obtained in study [21] and proves high precision of this technique.

In our experience, one of the disadvantages of this technique has been the difficulty determining the cutoff level (according to the Hounsfield scale) only for bone and cartilage structures at the stage of computer simulation. Degeneratively changed menisci are very often perceived by the software as parts of the tibial epiphysis even after hybrid parametric modeling and subsequently complicate intraoperative positioning of patient-specific guides. We believe that a combination of the patient's CT and MRI results is a solution to this problem.

\section{Conclusion}

Total knee arthroplasty using a virtual threedimensional prototype of the patient's lower limb and 3D printing of patient-specific guides allowed achieving good and excellent clinical and radiological results. Further improvement of positioning accuracy of knee endoprosthesis components using patient-specific precision guides requires upgrading the quality of computer simulation, combining static and dynamic 3D models, simultaneous CT and MRI data analysis, 
improving the methodology of rotational positioning of the components.

Study funding and conflict of interests. This study was not supported by any financial sources and the authors have no conflict of interests to disclose.

\section{References}

1. Kornilov N.N., Kulyaba T.A. Artroplastika kolennogo sustava [Arthroplasty of the knee joint]. Saint Petersburg: Sankt-Peterburgskiy nauchno-issledovatel'skiy institut travmatologii i ortopedii im. R.R. Vredena; 2012; 228 p.

2. Novoselov K.A., Kornilov N.N., Kulyaba T.A. Povrezhdeniya i zabolevaniya kolennogo sustava [Injuries and diseases of the knee joint]. Saint Petersburg: Gippokrat; 2006; $438 \mathrm{p}$.

3. Ignatenko V.L., Kornilov N.N., Kulyaba T.A., Selin A.V., Petukhov A.I., Croitoru I.I., Saraev A.V. Arthroplasty at valgus deformity of the knee (review). Travmatologiya $i$ ortopediya Rossii 2011; 4: 140-146.

4. Malyshev E.E., Pavlov D.V., Blinov S.V. Dynamic control of knee axial deformities. Travmatologiya $i$ ortopediya Rossii 2013; 3: 136-142.

5. Karyakin N.N., Malyshev E.E., Gorbatov R.O., Rotich G.K. 3D printing technique for patient specific instrumentation in total knee arthroplasty. Travmatologiya i ortopediya Rossii 2017; 23(3): 110-118. https://doi. org/10.21823/2311-2905-2017-23-3-110-118.

6. Wu L.D., Xiong Y., Yan S.G., Yang Q.S. Total knee replacement for posttraumatic degenerative arthritis of the knee. Chin J Traumatol 2005; 8(4): 195-199.

7. Malyshev E.E., Pavlov D.V., Gorbatov R.O. Total knee arthroplasty after proximal tibia fracture. Travmatologiya i ortopediya Rossii 2016; 1: 65-73.

8. Malyshev E.E., Zykin A.A., Gorbatov R.O., Kuvshinov S.G., Pavlov D.V. Mathematical modeling of load variation on the knee joint axial deformation in lower limb and results its clinical application. Sovremennye problemy nauki i obrazovaniya 2016; 2: 162.

9. Sereda A.P., Sagradyan A.S., Lychagin A.V. Patellar resurfacing of total knee arthroplasty. Kafedra travmatologii $i$ ortopedii 2012; 3: 18-28.

10. Yaffe M., Luo M., Goyal N., Chan P., Patel A., Cayo M., Stulberg S.D. Clinical, functional, and radiographic outcomes following total knee arthroplasty with patientspecific instrumentation, computer-assisted surgery, and manual instrumentation: a short-term follow-up study. Int $J$ Comput Assist Radiol Surg 2014; 9(5): 837-844, https://doi. org/10.1007/s11548-013-0968-6.

11. Voleti P.B., Hamula M.J., Baldwin K.D., Lee G.C. Current data do not support routine use of patient-specific instrumentation in total knee arthroplasty. J Arthroplasty 2014; 29(9): 1709-1712, https://doi.org/10.1016/j.arth.2014.01.039.

12. Hamilton W.G., Parks N.L., Saxena A. Patient-specific instrumentation does not shorten surgical time: a prospective, randomized trial. J Arthroplasty 2013; 28(8): 96-100, https:// doi.org/10.1016/j.arth.2013.04.049.

13. Nunley R.M., Ellison B.S., Ruh E.L., Williams B.M., Foreman K., Ford A.D., Barrack R.L. Are patient-specific cutting blocks cost-effective for total knee arthroplasty? Clin Orthop Relat Res 2012; 470(3): 889-894, https://doi. org/10.1007/s11999-011-2221-3.

14. Karyakin N.N., Gorbatov R.O. Patient specific instruments for total knee arthroplasty. Sovremennye problemy nauki i obrazovaniya 2016; 5: 23-33.

15. Baitov V.S., Mamuladze T.Z., Bazlov V.A. The possibility of using three-dimensional modeling and $3 D$ printing to create individual arthrodesis designs in revision arthroplasty of the knee joint. Mezhdunarodnyy zhurnal prikladnykh $i$ fundamental'nykh issledovaniy 2016; 12-7: 1189-1193.

16. Boonen B., Schotanus M.G., Kort N.P. Preliminary experience with the patient-specific templating total knee arthroplasty. Acta Orthop 2012; 83(4): 387-393, https://doi.org/ 10.3109/17453674.2012.711700.

17. Malyshev E.E., Pavlov D.V., Gorbatov R.O. Total knee arthroplasty after proximal tibia fracture. Travmatologiya $i$ ortopediya Rossii 2016; 1: 65-73.

18. Thienpont E., Grosu I., Paternostre F., Schwab P.E., Yombi J.C. The use of patient-specific instruments does not reduce blood loss during minimally invasive total knee arthroplasty? Knee Surg Sports Traumatol Arthrosc 2015; 23(7): 2055-2060, https://doi.org/10.1007/s00167-014-2952-2.

19. Heyse T.J., Tibesku C.O. Improved femoral component rotation in TKA using patient-specific instrumentation. Knee 2014; 21(1): 268-271, https://doi.org/10.1016/j. knee.2012.10.009.

20. Heyse T.J., Tibesku C.O. Improved tibial component rotation in TKA using patient-specific instrumentation. Arch Orthop Trauma Surg 2015; 135: 697-701, https://doi. org/10.1007/s00402-015-2157-2.

21. Noble J.W. Jr., Moore C.A., Liu N. The value of patient-matched instrumentation in total knee arthroplasty. J Arthroplasty 2012; 27(1): 153-155, https://doi.org/10.1016/j. arth.2011.07.006. 